\title{
THE METABOLISM OF VIRULENT HUMAN TUBERCLE BACILLI STUDIES IN ACID-FAST BACTERIA. XI.
}

Arthur I. Kendall, Alexander A. Day AND

Arthur W. WALKer

From the Patten Research Foundation, Northwestern University Medical School, Chicago

A study of the nitrogenous metabolism of certain rapidly growing acid-fast bacteria, ${ }^{1}$ so-called saprophytic human tubercle bacilli, in plain, glucose, mannitol and glycerol broths, revealed the fact that the organisms produced moderate amounts of ammonia, comparable quantitatively with that of the colon group, or approximately $30 \mathrm{mg}$. per $100 \mathrm{cc}$ of culture medium. ${ }^{2}$

The maximum production of ammonia was reached about the third week in the plain, glucose and mannitol cultures, the reaction becoming progressively alkaline during this period. In glycerol medium, on the other hand, the increase in ammonia was progressive to the fourth week, although the rate of production was slower and the reaction became acid, contrasting sharply in this respect with the alkalinity of the other cultures.

The ammonia curve gradually diminished from the maximum, particularly in plain glucose and mannitol mediums, until scarcely half the amount remained in solution at the end of the sixth week. The rise in ammonia coincides chronologically with the luxuriance of growth of the bacteria; the decline in ammonia is accompanied by recessive changes in the organisms, shown by a loss of staining intensity and the appearance of beaded forms.

A definite explanation for this phenomenon of waxing and waning of ammonia production is not apparent, but available evidence points to a coincidence between luxuriance of growth and ammonia increase (deamination) followed by autolysis of the organisms and a decrease in ammonia. Evidence in favor of this view is furnished by the rise

Received for publication Sept. 16, 1919.

1 Kendall, Day and Walker: Jour. Infect. Dis., 1914, 15, p. 417.

a Kendall, Day and Walker: Jour. Am. Chem. Soc., 1913, 35, p. 1201. 
and fall of esterase and lipase activity of the bacteria-free filtrates of these cultures as well as the esterase and lipase content of the bacteria themselves. ${ }^{3}$

Furthermore, total nitrogen determinations of the bacteria-free cultures show a small but unmistakable decrease at the height of bacterial development, indicating the incorporation of this element in the bacterial bodies, followed later by an increase in nitrogen, suggesting a gradual autolysis of the organisms with the liberation of some of the nitrogen in soluble form. ${ }^{4}$

The saprophytic human tubercle bacilli mentioned differ sharply from virulent human strains not only in their relative rapidity and luxuriance of growth in all ordinary mediums, but they fail to produce potent tuberculin even on prolonged cultivation. ${ }^{5}$

An important question, therefore, presents itself : In what way do virulent tubercle bacilli differ from avirulent types, and are there gradations in virulence among acid-fast organisms of the tubercle bacillus group?

In answering this question, it is of course necessary to keep constantly in view the probable difference in rates of chemical change which may result from difference in rate of development. A mere variation in the accumulation rate of the products of metabolism, coincident with corresponding relative luxuriance of growth of virulent and avirulent organisms, would be of far less significance than well defined differences in the types of reactions induced in parallel mediums.

A study was made of the nitrogenous metabolism of two virulent human tubercle bacilli, $A$ and $B$, each of which would kill a full-grown guinea-pig in from 3-4 weeks following the subcutaneous injection of $1 \mathrm{mg}$. of culture. Rabbits were unaffected by $10 \mathrm{mg}$. of culture. Each organism was inoculated into a sufficient number of $250 \mathrm{c} \mathrm{c}$ Erlenmeyer flasks (each containing $100 \mathrm{c} \mathrm{c}$ of $3 \%$ glycerol broth) so that 5 flasks could be studied in parallel at weekly intervals for a period of 8 weeks. The slow development of these two organisms make this plurality of parallel cultures desirable.

Evaporation is of necessity an important factor to control in prolonged cultivations because the free surface exposed, even in a moist incubator, will permit the loss of water, with a resulting confusion of results. Each flask, therefore, was incubated 24 hours prior to inoculation to insure sterility, and then weighed. Before analysis, the loss due to evaporation was compensated for by the addition of distilled water. By so doing, errors due to evaporation

S Kendall, Day and Walker: Jour. Infect. Dis., 1914, 15, p. 443.

- Kendall, Day and Walker: Ibid., 1914, 15, p. 433.

s Unpublished experiments by Arthur I. Kendall. 
were reduced to a minimum. Naturally, slight variations in the amount of evaporation between the different flasks occurred, but, on the whole, the results were surprisingly constant. The average replacement of water per flask at the end of the second week was $7.5 \mathrm{cc}$; the fourth week, $13.6 \mathrm{cc}$; the sixth week, $19 \mathrm{c} \mathrm{c}$, and the eighth week, $24 \mathrm{c} \mathrm{c}$.

The cultures in the various flasks grew at parallel rates. At the end of 2 weeks about $12 \%$ of the surface was covered with growth; 3 weeks, about $70 \%$, and at the 4 th week the bacilli formed a wrinkled, complete pellicle. The organisms were found to be completely acid-fast at the end of the 2nd week. By the 4th week, however, beaded forms began to appear, suggesting the beginning of recessive changes in the organisms.

The analyses comprised the determination of changes in the titratable reaction, using both neutral red and phenolphthalein, as indicators, the measurement of the ammonia, the total nitrogen and the amino-acid content of the bacteriafree medium.

A control was made from an uninoculated flask each week, and a composite sample, prepared from equal portions of each of the five flasks, was analyzed as well as the regular samples. The analyses of the individual flasks ran so closely parallel, the composite sample really added nothing to the definiteness of the data, and the results from it are omitted from the analytic data and the discussion.

The significance of a change in reaction, as a means for differentiating between human and bovine tubercle bacilli, has been studied by Theobald Smith and Marie Grund.' Generally speaking, the human type of culture in glycerol broth becomes acid; the bovine type, on the contrary, becomes progressively alkaline. Occasional variants intermediate between the two have been reported by Lewis ${ }^{8}$ and Grund. ${ }^{7}$

The significance of ammonia formation (deamination) as an index of the intracellular utilization of protein and protein derivatives has been emphasized in previous communications. ${ }^{2}$

The titration of amino-acids by the method of Sörenson is the only new analytic process introduced. The method followed was to add to $10 \mathrm{cc}$ of culture medium, exactly neutral to phenolphthalein, $5 \mathrm{cc}$ of exactly neutral liquor formaldehydi (using phenolphthalein as an indicator), and titrate with $\mathrm{N} / 50$ $\mathrm{NaOH}$. The determinations were made in duplicate and a variation of $0.1 \mathrm{cc}$ $\mathrm{N} / 50$ reagent was allowed as the greatest permissible deviation between two determinations from the same sample. Agreement within this limit was readily obtained.

Inasmuch as ammonia in the sample reacts with liquor formaldehydi, it is desirable to distinguish between total amino nitrogen and that portion which remains after ammonia is subtracted. The results in the following table are expressed as.milligrams of amino nitrogen minus ammonia per $100 \mathrm{c} c$ of sample.

Esterase and lipase determinations were made by the same methods as those previously described. ${ }^{3}$

The accompanying table contains the essential analytic data. The analyses of uninoculated control flasks, run in parallel with the inoculated flasks, are included to indicate the precision of the method nsed. It will be seen that the

- Jour. Med. Research, 1904-1905, 13, p. 405.

${ }^{7}$ Studies from the Research Laboratory, Department of Health, New York City, 1911, 6, p. 116

8 Jour. Exper. Med., 1910, 12, p. 82. 


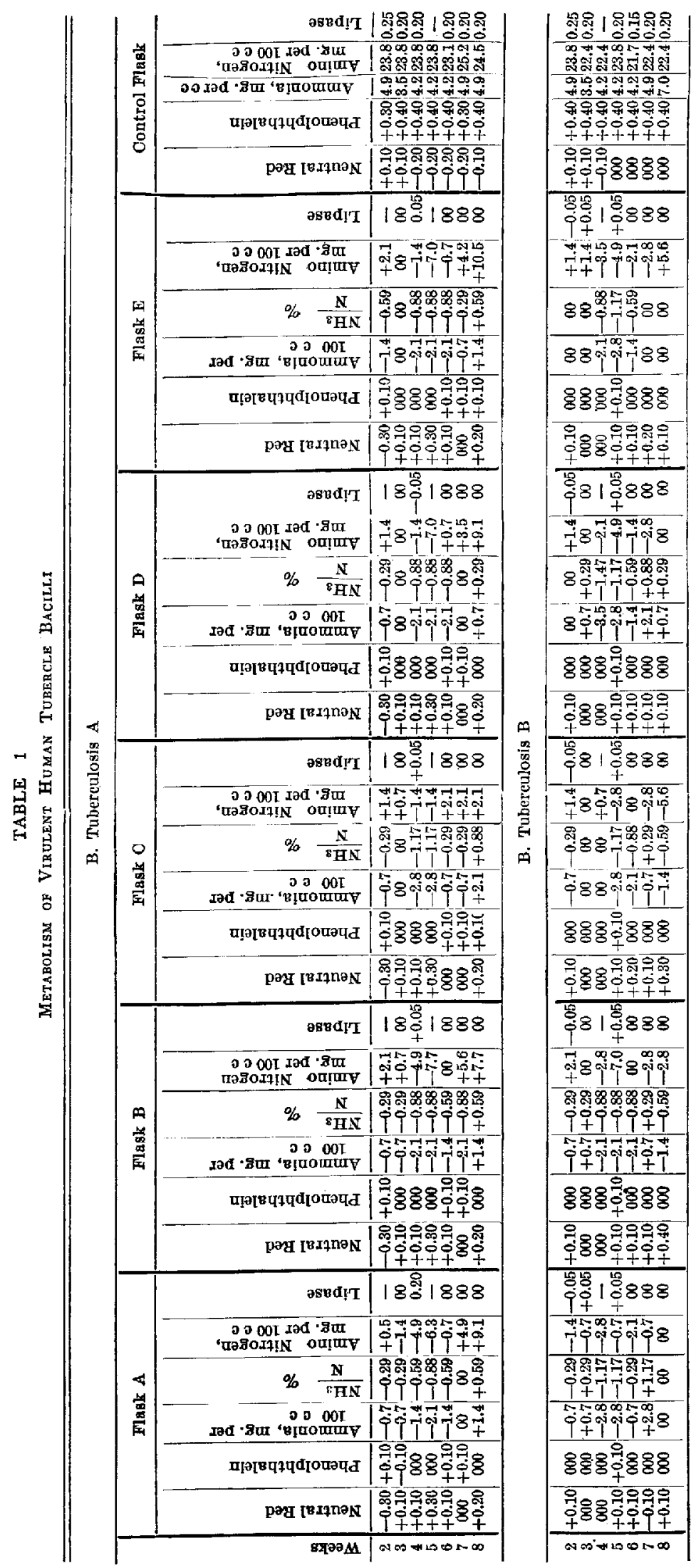


slight differences between parallel contrcl flasks for each week, and variations from week to week, are within the limits of error of the analytic procedures employed in this study.

DISCUSSION

Reaction.-Neutral red: The change in reaction is slight, but there is an unmistakable increase in titratable acidity from the first week of analysis.

Phenolphthalein: The change in reaction to phenolphthalein is less than that observed with neutral red, but it is toward the acid side.

The change in reaction from neutrality to acid is in accord with the general results of Theobald Smith and Marie Grund, and it is quite possible that the acidity is due in the last analysis to the utilization of glycerol for energy by the human types of tubercle bacilli.

Ammonia.-The ammonia curves for both strains are quantitatively very similar. There is a progressive decrease in the amount up to the end of the fourth week, then a gradual increase which reaches or even surpasses that found in the controls. Inasmuch as the decrease in ammonia parallels chronologically the period of increasing luxuriance of growth of the bacteria, it may be inferred that the two phenomena are inter-related. Conversely, the period of increasing soluble ammonia coincides in point of time with the recessive changes in the bacillary bodies, shown by the appearance in ever increasing numbers of beaded and feebly staining organisms. In other words, autolytic changes in the bacilli are accompanied by an increase in soluble ammonia.

Amino Nitrogen.-In general, the amino nitrogen curve follows the ammonia curve quite closely, but the excursion is somewhat greater, as might be expected. There is no evidence of proteolytic or enzymic action, however; otherwise, the amino nitrogen would tend to increase rather than diminish during the period of luxuriance of growth. Available evidence suggests that the decrease in ammonia and amino nitrogen is associated with the growth of the bacilli, and the gradual increase of these substances in solution, as the degenerative processes in the organisms become prominent, would be in harmony with this view.

Inasmuch as the final amount of amino nitrogen exceeds somewhat both that of the controls and of uninoculated broth at the start of the experiment, it would appear that the autolytic processes result in a liberation of amino-acids or polypeptids from the bacillary substance. 
Total Nitrogen.-A study of the total nitrogen in solution shows a gradual decrease which reaches a maximum at the height of vegetative activity of the organism, thus coinciding approximately with the corresponding period of greatest decrease both in ammonia and in amino nitrogen. The amount of decrease in total nitrogen is greater than the sums of the ammonia and amino nitrogen at this time, which is in harmony with the obvious incorporation of this element in the substance of the bacilli. The gradual increase in soluble total nitrogen, as autolysis proceeds, is in accord with the observations recorded above.

Esterase and Lipase.-A study of the esterase and lipase content of the mediums was made, using the methods described in previous communications. $^{3}$ Both the esterase and lipase content of the culture medium was practically nil, contrasting sharply in this respect with corresponding observations in cultures of rapidly growing "saprophytic" tubercle bacilli, previously studied. This observation is in harmony with that of Wells and Corper, ${ }^{\circ}$ who found that the lipase activity of tubercle bacilli was decidedly less pronounced than that of staphylococci and Bacillus pyocyaneus. In this respect, the virulent human tubercle bacilli studied differ sharply from so-called avirulent types.

\section{SUMMARY}

Parallel cultures of virulent human tubercle bacilli, grown under the same conditions in glycerol broth, develop with remarkably uniform luxuriance, and the chemical changes associated with this development are quantitatively and qualitatively very similar.

The noteworthy chemical features are the production of a feebly acid reaction, together with a gradual decrease in ammonia, amino nitrogen and total nitrogen in solution up to the point of maximum development of the culture. As the recessive changes, resulting presumably in autolysis of the bacilli, become dominant, a resolution of nitrogeneous constituents becomes manifest. This proceeds until a very considerable return to conditions in the original quantitative nitrogenous composition of the culture medium is attained. The amino nitrogen, however, is somewhat greater than the original content, suggesting that the autolytic process yields considerable amounts of nitrogenous substance, which can be measured by the Sörenson method of formol titration.

- Jour. Infect. Dis., 1912, 11, p. 388. 
The contrast between the development of an acid reaction, on the one hand, and the minimal evidence of deamination (shown by the ammonia curve) and of action on the protein constituents of the medium (amino nitrogen production), on the other hand, suggests that glycerol may be utilized largely for the energy requirements of the bacilli, thus shielding the protein constituents from extensive breakdown. In other words, the glycerol appears to exert a sparing action for the protein constituents of the medium. This is in harmony with the generally accepted view that glycerol is a desirable, if not essential, constituent of medium suitable for the cultivation of the human types of the tubercle bacillus.

It is in sharp contrast to the bovine types of tubercle bacilli, which do not appear to require the addition of glycerol as a constituent of mediums adapted to their cultivation. 